\title{
Nonlinear fractional waves at elastic interfaces
}

\author{
Julian Kappler, ${ }^{1}$ Shamit Shrivastava, ${ }^{2}$ Matthias F. Schneider, ${ }^{3}$ and Roland R. Netz ${ }^{1}$ \\ ${ }^{1}$ Department of Physics, Freie Universität Berlin, 14195 Berlin, Germany \\ ${ }^{2}$ Institute of Biomedical Engineering, University of Oxford, Oxford OX3 7DQ, United Kingdom \\ ${ }^{3}$ Department of Physics, Technische Universität Dortmund, 44227 Dortmund, Germany
}

(Received 12 February 2017; published 20 November 2017)

\begin{abstract}
We derive the nonlinear fractional surface wave equation that governs compression waves at an elastic interface that is coupled to a viscous bulk medium. The fractional character of the differential equation comes from the fact that the effective thickness of the bulk layer that is coupled to the interface is frequency dependent. The nonlinearity arises from the nonlinear dependence of the interface compressibility on the local compression, which is obtained from experimental measurements and reflects a phase transition at the interface. Numerical solutions of our nonlinear fractional theory reproduce several experimental key features of surface waves in phospholipid monolayers at the air-water interface without freely adjustable fitting parameters. In particular, the propagation distance of the surface wave abruptly increases at a threshold excitation amplitude. The wave velocity is found to be of the order of $40 \mathrm{~cm} / \mathrm{s}$ in both experiments and theory and slightly increases as a function of the excitation amplitude. Nonlinear acoustic switching effects in membranes are thus shown to arise purely based on intrinsic membrane properties, namely, the presence of compressibility nonlinearities that accompany phase transitions at the interface.
\end{abstract}

DOI: 10.1103/PhysRevFluids.2.114804

\section{INTRODUCTION}

Surface waves are waves that are localized at the interface between two media and are at the core of many important everyday life phenomena [1-6]. As a consequence of energy conservation and the interfacial localization, and neglecting dissipative damping effects, the intensity of a surface wave excitation at a planar interface originating from a point source falls off with the inverse distance and not with the inverse squared distance, as for ordinary bulk waves. Consequently, in the absence of viscous effects, a surface wave emanating from a line excitation travels basically without attenuation. This demonstrates that surface waves dominate over regular bulk waves at large enough distance and thus explains why they have been amply studied experimentally and theoretically [7-22]. For different systems one finds distinct surface wave types. At the interface between two fluids that have different densities, one finds capillary-gravity waves, the best-known realization of which are deep-water waves at the air-water interface [8]. Depending on the wavelength, these waves are dominated either by gravity or by the interfacial tension. From measurements of the dispersion relation, the functional relationship between wavelength and frequency, fluid [23], and interfacial properties [24] can be extracted. At the surface of an elastic solid one finds Rayleigh waves, with a dispersion relation that depends on the viscoelastic modulus of the solid $[9,11,12,25]$. Rayleigh and capillary-gravity waves are distinct surface wave types that in fact can, for suitably chosen material parameters, coexist [25]. Since they are linear phenomena, i.e., described by a theory that is linear in the surface wave amplitude, they are predicted to travel independently from each other even if they are excited at the same frequency or the same wavelength. If the interface in addition to tension exhibits a finite compressibility, a third surface wave type exists, referred to as a Lucassen wave $[16,17,19]$. A well-studied experimental realization is a monolayer of amphiphilic molecules at the air-water interfaces $[15-17,19,21,26]$. At the experimentally relevant low-frequency range and for realistic values of the interfacial elastic modulus [27], Lucassen waves exhibit wavelengths in the centimeter range and are thus easily excitable and observable in typical experiments with self-assembled monolayers [17,21]. 
Waveguiding phenomena in monolayers have recently received focal attention because of the possible connection to nerve-pulse propagation [28-33], cell-membrane-mediated acoustic cell communication [28,34-36], and pressure-pulse-induced regulation of membrane protein function $[29,37,38]$. One exciting recent finding was the discovery of nonlinear wave switching phenomena in a simple system of a dipalmitoylphosphatidylcholine (DPPC) lipid monolayer spread on the air-water interface [29]. In the experiments, the wave propagation speed and the wave attenuation were demonstrated to depend in a highly nonlinear fashion on the excitation amplitude, showing almost all-or-nothing behavior: Only above a certain threshold of the excitation amplitude does wave propagation set in, while below that threshold wave transmission is experimentally almost negligible [29]. Such a nonlinear switching phenomenon offers a multitude of exciting applications and interpretations, in particular since it has been known for a long time that nerve pulse propagation is always accompanied by a mechanical displacement traveling in the axon membrane [30,39-41]. In that connection, it should be noted that many membrane proteins are pressure sensitive $[37,38]$, so the existence of nonlinear acoustic phenomena in membranes constitutes an exquisite opportunity for smart membrane-based regulation and information processing applications $[36,42,43]$.

The theoretical description of such nonlinear surface wave phenomena is challenging for several reasons. First of all, the dispersion relation between wave frequency $\omega$ and wave number $k=2 \pi / \lambda$ that describes small-amplitude linear surface waves can generally be written as

$$
k^{2} \sim \omega^{\alpha},
$$

where we define the dispersion exponent $\alpha$ that allows one to classify surface wave equations. For normal compression waves one has $\alpha=2$ and thus the frequency is linearly related to the wave vector. However, for surface waves one typically finds $\alpha \neq 2$. For gravity waves $\alpha=4$, for capillary waves $\alpha=4 / 3$, and for Lucassen waves one has $\alpha=3 / 2$ [16,44].

Nonlinear wave effects (i.e., effects that are nonlinear in the wave amplitude) cannot be simply added on the level of a dispersion relation, since a dispersion relation is obtained by Fourier transforming a linear wave equation and by construction is restricted to the linear regime. Rather, nonlinear effects in the wave amplitude are only captured by a properly derived nonlinear differential equation in terms of the local perturbation field that describes the microscopic wave propagation. This is why in previous theoretical treatments of nonlinear surface waves, the starting point was typically the standard wave equation with $\alpha=2$ and nonlinear effects were introduced phenomenologically $[31,35,45]$. It is altogether not clear whether this constitutes an accurate theoretical framework for the description of nonlinear surface compression waves, which Lucassen predicted to have $\alpha=3 / 2$. On the other hand, hitherto no real-space differential equation for the Lucassen dispersion relation had been derived.

In this article we first derive the linear real-space equation that describes Lucassen surface waves from standard hydrodynamics. We show that these waves are described by a so-called fractional wave equation, which is a differential equation with a fractional, i.e., noninteger, time derivative. Although linear fractional wave equations have been amply described in the literature [46-52], until now no derivation of such an equation based on physical first principles had been available.

In a second step, we also include nonlinear effects in the wave amplitude by accounting for the nonlinear interfacial compressibility. The necessary material parameters are taken from our experimental measurements of the interfacial compressibility of DPPC monolayers at the air-water interface. We show that nonlinear effects become dominant for monolayers close to a phase transition, where the two-dimensional elastic modulus (the inverse compressibility) becomes small or even vanishes, thus explaining previous experimental observations [29]. We solve our nonlinear fractional wave equation numerically and calculate the wave velocity and the compression amplitude as a function of the excitation amplitude. In agreement with experimental observations [29], we find an abrupt decrease of wave damping accompanied by a mild increase in wave velocity above a threshold excitation amplitude. In this comparison, no fitting parameter is used; rather, we extract the nonlinear monolayer compressibility and all other parameters from our experimental measurements. 
Our results show that acoustic phenomena at self-assembled phospholipid monolayers are quantitatively described by a nonlinear fractional wave equation derived from physical first principles. Since phospholipids at typical surface pressures are quite close to a phase transition accompanied by an anomalously high interfacial compressibility [53], nonlinear effects are substantial and lead to a nonlinear dependence of the wave propagation properties on the excitation amplitude. This not only shows that phospholipid layers can guide the propagation of acoustic waves, but they can also process these waves in a nonlinear fashion. In this context it is interesting to note that biological membranes are actively maintained at a state close to a membrane phase transition $[36,53,54]$, so this nonlinear switching phenomenon could possibly play a crucial role in the communication between pressure-sensitive membrane proteins and other functional units situated in membranes. The resulting acoustic wave speed close to the threshold excitation amplitude is found to be about $40 \mathrm{~cm} / \mathrm{s}$ in both experiments and theory. Remarkably, this speed is thus in a range comparable to the action potential speed in nonmyelinated axons [55-58]. The present work should be viewed as a fundamental step in understanding the relation between the acoustic nonlinear membrane wave, treated in this article, and the electrochemically generated action potential, described by the nonlinear Hodgkin-Huxley equations [59].

The structure of this article is as follows. We first sketch the derivation of the dispersion relation for Lucassen waves using linearized theory. We then convert this dispersion relation into a corresponding fractional wave equation. We present a simple physical interpretation of the fractional derivative that appears in the differential equation in terms of the frequency-dependent coupling range of the surface wave to the underlying bulk fluid. It is important to note that the linear fractional wave equation is also systematically derived from interfacial momentum conservation, which is detailed in the Supplemental Material (SM) [60]. In a second step we include nonlinear effects by accounting for the change of the monolayer compressibility due to the local monolayer density change that accompanies a finite-amplitude surface wave. The resulting nonlinear fractional wave equation is numerically solved in an interfacial geometry that closely mimics the experimental setup used to study surface waves in monolayers at the air-water interface [29]. Finally, we compare numerical predictions for the wave velocity and the wave damping with experimental results. This comparison is done without any fitting parameters, as all model parameters are extracted from experiments. The experimental wave speed of about $40 \mathrm{~cm} / \mathrm{s}$ is very accurately reproduced by the theory. We also reproduce the sudden change of the surface wave propagation properties at a threshold excitation amplitude and thus explain the nonlinear surface wave behavior in terms of the compressibility nonlinearity of a lipid monolayer.

\section{DERIVATION OF THE NONLINEAR FRACTIONAL SURFACE WAVE EQUATION}

\section{A. Dispersion relation for Lucassen surface waves}

We here recapitulate the main steps in the derivation of the Lucassen dispersion relation $[15,16,19]$; complete details can be found in the SM [60]. We consider a semi-infinite incompressible Newtonian fluid in the half space $z \leqslant 0$ with shear viscosity $\eta$ and mass density $\rho$, covered by an interface at $z=0$ with two-dimensional excess mass density $\rho_{2 \mathrm{D}}$, and which responds elastically under compression, with elastic modulus (inverse compressibility) $K_{2 \mathrm{D}}$ [15-17,19] (see Fig. 1). We neglect interfacial excess viscosity $[61,62]$ and bending rigidity $[63,64]$ effects in this work, which could easily be included in the derivation $[60,65]$.

We start with the linearized incompressible Navier-Stokes equation in the absence of external forces [44]

$$
\rho \frac{\partial \vec{v}(\vec{r}, t)}{\partial t}=-\vec{\nabla} P(\vec{r}, t)+\eta \vec{\nabla}^{2} \vec{v}(\vec{r}, t)
$$

where $\vec{v}(\vec{r}, t)$ is the vectorial velocity field and $P(\vec{r}, t)$ is the pressure field. The gradient operator is defined as $\vec{\nabla}=(\partial / \partial x, \partial / \partial y, \partial / \partial z)$, where the Cartesian coordinates are defined as $\vec{r}=(x, y, z)$. Note that in the linearized Navier-Stokes equation (2) we have neglected the convective term nonlinear 


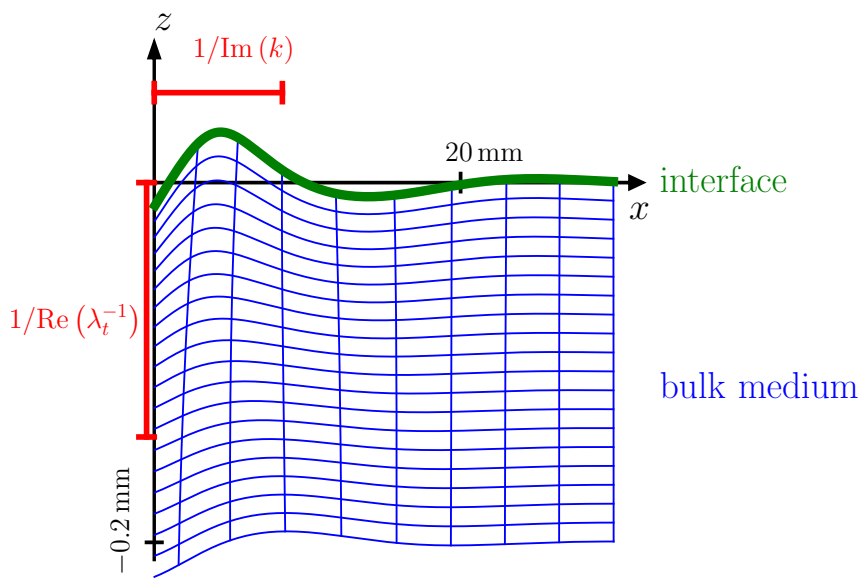

FIG. 1. Displacement field of the Lucassen wave, given by Eqs. (4), (8), and (9). The decay lengths in both the $x$ and $z$ directions are shown in red, with $k$ and $\lambda_{t}$ given by Eqs. (14) and (13), respectively. For the bulk medium, water is used $\left(\rho=10^{3} \mathrm{~kg} / \mathrm{m}^{3}\right.$ and $\left.\eta=10^{-3} \mathrm{Pas}\right)$; the interface parameters are chosen appropriately for a DPPC monolayer $\left(K_{2 \mathrm{D}}=10 \mathrm{mN} / \mathrm{m}\right.$ and $\left.\rho_{2 \mathrm{D}}=10^{-6} \mathrm{~kg} / \mathrm{m}^{2}\right)$. The shown solution has a frequency $\omega=100 \mathrm{~s}^{-1}$. Note the anisotropic scaling in the $x$ and $z$ directions and furthermore that the units in a similar plot in Ref. [25] are wrong.

in the velocity field. This approximation is valid since, as we show in detail in the SM [60], the nonlinear effects due to surface compression we will consider later on are much stronger than this convective term. Relating the velocity field to the time derivative of the displacement field $\vec{u}(\vec{r}, t)$ as

$$
\vec{v}(\vec{r}, t)=\partial \vec{u}(\vec{r}, t) / \partial t
$$

and decomposing the displacement field into the longitudinal and transversal parts according to

$$
\vec{u}(\vec{r}, t)=\vec{\nabla} \Phi(\vec{r}, t)+\vec{\nabla} \times \vec{\Psi}(\vec{r}, t),
$$

one finds that the incompressibility condition $\vec{\nabla} \cdot \vec{v}(\vec{r}, t)=0$ and the linearized Navier-Stokes equation (2) can be rewritten as

$$
\begin{gathered}
\vec{\nabla}^{2} \Phi(\vec{r}, t)=0, \\
\eta \vec{\nabla}^{2} \vec{\Psi}(\vec{r}, t)=\rho \partial \vec{\Psi}(\vec{r}, t) / \partial t .
\end{gathered}
$$

Likewise, the pressure profile follows as

$$
P(\vec{r}, t)=-\rho \partial^{2} \Phi(\vec{r}, t) / \partial t^{2} .
$$

To solve Eqs. (5) and (6) for a wave of frequency $\omega$ and wave number $k$ that is localized in the $x y$ plane and travels along the $x$ direction, we make the harmonic wave ansatz [16]

$$
\begin{gathered}
\Phi(\vec{r}, t)=\phi e^{z / \lambda_{l}} e^{i(k x-\omega t)}, \\
\vec{\Psi}(\vec{r}, t)=\hat{e}_{y} \psi e^{z / \lambda_{t}} e^{i(k x-\omega t)},
\end{gathered}
$$

where the prefactors $\phi$ and $\psi$ are the wave amplitudes and $\hat{e}_{y}$ is the unit vector in the $y$ direction. The decay lengths $\lambda_{l}$ and $\lambda_{t}$ describe the exponential decay of the longitudinal and transversal parts 
away from the interface (in the $z$ direction) and follow from Eqs. (5) and (6) as

$$
\begin{aligned}
& \lambda_{l}^{-2}=k^{2}, \\
& \lambda_{t}^{-2}=k^{2}+\frac{-i \omega \rho}{\eta} .
\end{aligned}
$$

The ratio of the wave amplitudes $\phi$ and $\psi$ is fixed by the stress continuity boundary condition at the surface $z=0$, which gives rise to a rather complicated dispersion relation (see the SM for a full derivation [60]). In the long-wavelength limit, defined by the condition $\rho \omega \gg \eta k^{2}$, this dispersion relation simplifies to

$$
k^{2}=\frac{\omega^{2}}{K_{2 \mathrm{D}}}\left(\rho_{2 \mathrm{D}}+\rho \lambda_{t}\right)
$$

as derived in the SM [60]. In the same long-wavelength limit $\rho \omega \gg \eta k^{2}$, the expression for the transversal decay length (11) simplifies to

$$
\lambda_{t}=\sqrt{\frac{\eta}{-i \omega \rho}},
$$

so we finally obtain, by combining Eqs. (12) and (13), the Lucassen dispersion relation

$$
k^{2}=\frac{\omega^{2}}{K_{2 \mathrm{D}}}\left(\sqrt{\frac{i \rho \eta}{\omega}}+\rho_{2 \mathrm{D}}\right) .
$$

This expression in fact constitutes a slight generalization of the standard Lucassen dispersion relation [16] as it additionally contains the interfacial excess mass density $\rho_{2 \mathrm{D}}$ [25]. This generalized dispersion relation is very useful for our discussion, since it allows us to distinguish two important physical limits: In case the coupling to the subphase vanishes, which can be achieved by sending either the bulk viscosity $\eta$ or the bulk density $\rho$ to zero, the first term on the right-hand side of Eq. (14) vanishes. In this limit we are left with the standard dispersion relation for an elastic wave that involves the elasticity and mass density parameters $K_{2 \mathrm{D}}$ and $\rho_{2 \mathrm{D}}$ of the interface. On the other hand, if the interfacial excess mass is neglected, i.e., for $\rho_{2 \mathrm{D}}=0$, the classical Lucassen dispersion relation is obtained from Eq. (14). A simple physical interpretation of Eq. (14) will be presented in the next section.

\section{B. Linear fractional differential equation for Lucassen surface waves}

We now give a simple heuristic derivation of the linear fractional wave equation corresponding to the Lucassen wave. In the SM [60], we provide a rigorous derivation based on momentum conservation and utilizing the stress continuity boundary conditions at the interface.

The key observation for arriving at a linear fractional wave equation is that the generalized Lucassen dispersion relation (14) can be rewritten as

$$
(i k)^{2} K_{2 \mathrm{D}}=(-i \omega)^{2} \rho_{2 \mathrm{D}}+(-i \omega)^{3 / 2} \sqrt{\rho \eta}
$$

or, using the approximate expression for the longitudinal decay length $\lambda_{t}$, which characterizes the vertical decay of the surface wave [60], Eq. (13), as

$$
(i k)^{2} K_{2 \mathrm{D}}=(-i \omega)^{2}\left(\rho_{2 \mathrm{D}}+\lambda_{t} \rho\right) .
$$

The latter equation allows for a simple physical interpretation: The effective area mass density of the interface is given by the sum of the interfacial excess mass density $\rho_{2 \mathrm{D}}$ and the area mass density of the bulk fluid layer that via viscosity is coupled to the interface. The area mass density of the coupled bulk fluid layer is $\lambda_{t} \rho$, which is the product of the surface wave decay length $\lambda_{t}$ and the bulk mass density $\rho$. The fractional exponent in Eq. (15) emerges because the decay length $\lambda_{t}$ in Eq. (13) 
depends as an inverse square root on the wave frequency $\omega$, reflecting that lower frequencies reach deeper into the fluid bulk medium.

Since Eq. (15) was derived from the harmonic wave ansatz (8) and (9), it is not straightforward to interpret it is as the Fourier transform of a one-dimensional displacement field. However, as we detail in the SM [60], for the Lucassen wave, the displacement in the $z$ direction is much smaller than the displacement in the $x$ direction, which allows us to interpret Eq. (15) as the Fourier transform of the fractional differential wave equation

$$
K_{2 \mathrm{D}} \frac{\partial^{2} U(x, t)}{\partial x^{2}}=\rho_{2 \mathrm{D}} \frac{\partial^{2} U(x, t)}{\partial t^{2}}+\sqrt{\rho \eta} \frac{\partial^{3 / 2} U(x, t)}{\partial t^{3 / 2}},
$$

acting on the displacement of the interface in the $x$ direction, i.e., along the surface, which we define as $U(x, t)=u_{x}(x, z=0, t)$. As in the derivation of Eq. (15), the displacement field $U(x, t)$ is independent of $y$, we are thus considering a surface wave front that travels in the $x$ direction and that is translationally invariant in the $y$ direction. While we derive Eq. (17) based on the dispersion relation (15) and the properties of the Lucassen wave solution, it is also possible to derive the fractional wave equation (17) directly from the stress boundary condition at the interface $z=0$ (see the SM for details [60]). Furthermore, it is possible to recover the interfacial displacement in the $z$ direction from a solution of Eq. (17) [60]. The fractional derivative $\partial^{3 / 2} / \partial t^{3 / 2}$ on the right-hand side of Eq. (17) is defined in Fourier space, where it amounts to multiplication by $(-i \omega)^{3 / 2}[46,47]$. In real space, the fractional derivative in Eq. (17) can be formulated using the Caputo formula $[46,66]$

$$
\frac{\partial^{3 / 2} U(x, t)}{\partial t^{3 / 2}}=\frac{1}{\sqrt{\pi}} \int_{0}^{t}(t-s)^{-1 / 2} \frac{\partial^{2} U(x, s)}{\partial s^{2}} d s,
$$

which holds for times $t \geqslant 0$ and where we assume the interface to be in equilibrium at $t=0$ so that both $U(x, t)$ and $\partial U(x, t) / \partial t$ vanish for $t<0$. Thus, Eq. (17) is actually an integro-differential equation that is nonlocal in time. The non-Markovian nature of Eq. (17) has an intuitive interpretation: It is well known that eliminating degrees of freedom from a dynamical system leads to non-Markovian equations [67,68]; the integral appearing in Eq. (18) can thus be thought of as a consequence of eliminating the displacement field of the bulk medium at $z<0$ from the dynamics.

For a DPPC monolayer on water we have a typical interfacial excess mass density $\rho_{2 \mathrm{D}}=$ $10^{-6} \mathrm{~kg} / \mathrm{m}^{2}$ [28], the bulk water mass density is $\rho=10^{3} \mathrm{~kg} / \mathrm{m}^{3}$, and the viscosity of water is $\eta=10^{-3} \mathrm{Pas}$ [69]. It follows that for frequencies $\omega \lesssim 10^{7} \mathrm{~s}^{-1}$, the effects due to the membrane mass $\rho_{2 \mathrm{D}}$ in Eq. (15) are negligible compared to the water layer mass. Thus we will for our comparison with experimental data neglect the membrane excess mass term proportional to $\rho_{2 \mathrm{D}}$ in Eq. (17) in the following. We note that the resulting linear fractional wave equation has been studied in detail and in fact analytical solutions are well known [46,60,70,71], which we use to test our numerical implementation. For the nonlinear fractional wave equation that we derive in the next section no analytical solutions are known, so it must be solved numerically.

\section{Nonlinear compressibility effects}

The isothermal elastic modulus $K_{2 \mathrm{D}}$ of a lipid monolayer at the air-water interface follows from the surface pressure isotherm $\pi(a)$ as [72]

$$
K_{2 \mathrm{D}}=-\left.a \frac{\partial \pi(a)}{\partial a}\right|_{T},
$$

where $a$ is the area per lipid. An experimentally measured isotherm $\pi(a)$ for a DPPC monolayer at room temperature is shown in the inset of Fig. 2; the resulting elastic modulus $K_{2 \mathrm{D}}$ according to Eq. (19) follows by numerical differentiation and is shown in Fig. 2 by a solid line. Note that lipid molecules are essentially insoluble in water, so the number of lipid molecules in the monolayer at the air-water interface stays fixed as the surface pressure is changed, which is why a finite equilibrium compressibility is obtained; such a monolayer is called a Langmuir monolayer. In Fig. 2 it can 


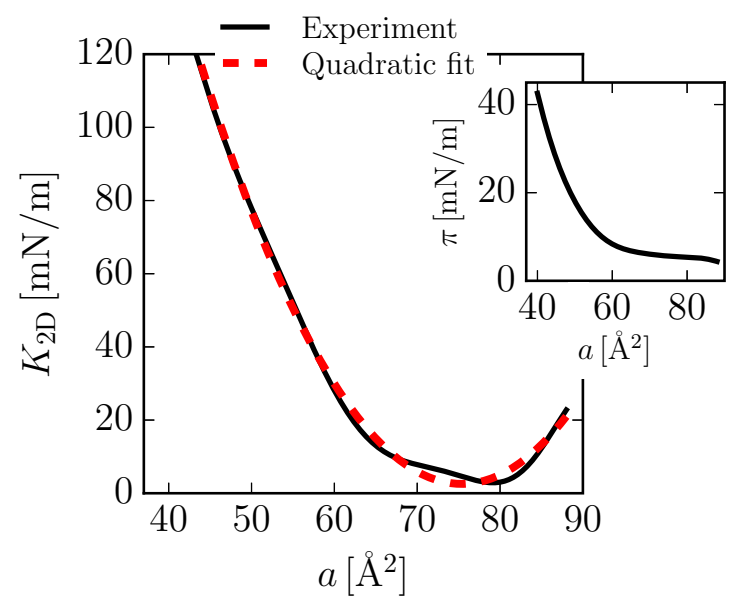

FIG. 2. Langmuir isotherm and corresponding isothermal elastic modulus. The inset shows an experimentally measured pressure-area isotherm for a (Langmuir) DPPC monolayer [73]. The main plot shows the corresponding isothermal elastic modulus $K_{2 \mathrm{D}}$, as calculated from Eq. (19) using the isotherm from the inset. The red dashed line shows a quadratic polynomial fit to the elastic modulus.

be seen that the modulus $K_{2 \mathrm{D}}$ depends sensitively on the area per lipid molecule $a$ and exhibits a minimum at an intermediate value of the area. This minimum signals a smeared-out surface phase transition, at which the area per lipid $a$ changes drastically as the surface pressure $\pi$ is varied, as can be clearly seen in the inset of Fig. 2 . The overall area dependence of the area modulus $K_{2 \mathrm{D}}$ can be well represented by a second-order polynomial fit to the experimental data,

$$
K_{2 \mathrm{D}}=K_{2 \mathrm{D}}^{(0)}+K_{2 \mathrm{D}}^{(2)}\left(a-a_{0}\right)^{2},
$$

which is shown as a red dashed line in Fig. 2. The fit values we extract from our experimental data are $K_{2 \mathrm{D}}^{(0)}=2.55 \mathrm{mN} / \mathrm{m}, a_{0}=75.4 \AA^{2}$, and $K_{2 \mathrm{D}}^{(2)}=0.12 \mathrm{mN} / \AA^{2}$.

The linear wave equation (17) assumes that the local change of the area per lipid during wave propagation is small, so the elastic modulus $K_{2 \mathrm{D}}$ does not change appreciably. This approximation is valid for small wave amplitudes, but for large enough amplitudes the wave will cause local changes in $K_{2 \mathrm{D}}$ that cannot be neglected in Eq. (17). For a one-dimensional surface wave characterized by the in-plane displacement field $U(x, t)$, the local time-dependent area per lipid is related to the divergence of the displacement field via [72]

$$
a(x, t)=\bar{a}\left(1+\frac{\partial U(x, t)}{\partial x}\right)
$$

where $\bar{a}$ denotes the equilibrium area per lipid in the absence of the surface wave.

Inserting the expression (21) for the space- and time-dependent area $a(x, t)$ into the parabolic approximation for the elastic modulus (20), we obtain

$$
K_{2 \mathrm{D}}=K_{2 \mathrm{D}}^{(0)}+K_{2 \mathrm{D}}^{(2)}\left(\bar{a}+\bar{a} \frac{\partial U(x, t)}{\partial x}-a_{0}\right)^{2},
$$

which constitutes a relation between the local elastic modulus $K_{2 \mathrm{D}}$ and the interfacial displacement field $U(x, t)$. In deriving this relation, we assume that the experimental isotherm in Fig. 2, which is obtained from an equilibrium experiment where the entire monolayer is uniformly compressed at fixed temperature, also describes the local time-dependent elastic response of the monolayer at the typical length and time scales of a propagating surface wave. The question whether the measured isotherm is appropriate for the time scales we consider will be discussed later; for now we show that 
it is applicable at the length scales of interest: The typical surface wavelengths $\lambda=2 \pi / k$ are, in the experimentally relevant frequency range $\omega$ from 1 to $10^{6} \mathrm{~Hz}$, in the range of tens of centimeters down to $0.1 \mathrm{~mm}$, as follows directly from the Lucassen dispersion relation (15); they are therefore much larger than the lipid size $\sim \sqrt{a}$ and the locality approximation is not expected to lead to any problems. So we conclude that the expression for the local isothermal elastic modulus (22) is valid to leading order at the length scales of interest.

Combining the displacement-dependent expression for the elastic elastic modulus (22) with the fractional wave equation (17), we finally obtain

$$
\left[K_{2 \mathrm{D}}^{(0)}+\bar{a}^{2} K_{2 \mathrm{D}}^{(2)}\left(1+\frac{\partial U(x, t)}{\partial x}-\frac{a_{0}}{\bar{a}}\right)^{2}\right] \frac{\partial^{2} U(x, t)}{\partial x^{2}}=\sqrt{\rho \eta} \frac{\partial^{3 / 2} U(x, t)}{\partial t^{3 / 2}},
$$

where, as discussed after Eq. (18), we neglect the inertial term proportional to the membrane mass density $\rho_{2}$ D. This nonlinear fractional wave equation constitutes the central result of our paper. A few comments on the approximations involved and the limits of applicability are in order.

(i) We emphasize in our derivation that the displacement $U(x, t)$ is so small that the linearized Navier-Stokes equation (2) is valid, while at the same time $U(x, t)$ is large enough that the assumption of a constant elastic modulus $K_{2 \mathrm{D}}$ breaks down. In essence, Eq. (23) is valid and relevant for an intermediate range of displacement amplitudes. In the SM we show that this assumption is indeed appropriate for the experiments we are comparing with further below and also discuss at which amplitudes nonlinear effects become relevant $[60,74]$. In short, this happens once the spatial derivative of $U(x, t)$ is so large that $K_{2 \mathrm{D}}$ as defined in Eq. (22) can no longer be approximated as constant.

(ii) Note that when the elastic modulus $K_{2 \mathrm{D}}$ depends on the displacement field $U(x, t)$, as demonstrated in Eq. (22), it makes a difference whether $K_{2 \mathrm{D}}$ appears in front, in between, or after the two spatial derivatives in Eq. (17). In our nonlinear Eq. (23), $K_{2 \mathrm{D}}$ is positioned in front of the spatial derivatives, so the derivatives do not act on $K_{2 \mathrm{D}}$. This structure of the equation is rigorously derived in the SM [60].

(iii) The explicit values for the coefficients appearing in the parabolic fit of the experimental elastic modulus in Eq. (20) are taken from the equilibrium measurement shown in Fig. 2; these values thus correspond to an isothermal measurement at fixed temperature. In the SM we show that the elastic modulus appropriate for small-amplitude Lucassen waves is expected to be somewhat between isothermal and adiabatic, as the time scale of heat transport into the bulk medium is comparable to the oscillation time [60]. For large wave amplitudes the heat produced or consumed during expansion and compression is therefore not transported into the bulk fluid quickly enough, so the temperature locally deviates from the environment. For large wave amplitudes the interface deformation is thus expected to become rather adiabatic. The details of this depends on material parameters such as the monolayer heat conductivity and heat capacity, which are not well characterized experimentally. We thus perform our actual numerical calculations with the isothermal values extracted from Fig. 2, bearing in mind that this is clearly an approximation.

\section{NUMERICAL SOLUTION}

We numerically solve Eq. (23) in the finite spatial domain $x \in[0, L]$ with the initial condition

$$
U(x, t=0)=\frac{\partial U(x, t=0)}{\partial t}=0
$$

for all $x$, corresponding to an initially relaxed and undeformed membrane and the boundary conditions

$$
\begin{aligned}
U(x=0, t) & =U_{0}(t), \\
U(x=L, t) & =0 .
\end{aligned}
$$


The function $U_{0}(t)$ in Eq. (25) models the mechanical monolayer excitation at the left boundary $x=0$, which experimentally is produced by a moving piezodriven blade that is in direct contact with the monolayer at the interface (see Ref. [29] for more experimental details). The boundary condition (26) mimics the effects of a bounding wall with vanishing monolayer displacement at a distance $L$ from the excitation source.

We solve the boundary-value problem defined by Eqs. (23)-(26) by a modification of a general numerical scheme for nonlinear fractional wave equations [75]. In the numerics we discretize the equations on 300 grid points and use a system size of $L=3 \mathrm{~cm}$, which is demonstrated to be large enough so that finite-size effects in the observables we consider can be neglected [60]. The accuracy of our numerical scheme is demonstrated by comparison with analytical solutions that are available for the linear fractional wave equation (17) $[46,70,71,76]$. Details of our numerical implementation can be found in the SM [60].

For the mechanical boundary excitation $U_{0}(t)$ we use a smoothed pulse function of the form

$$
U_{0}(t)=U_{0}^{\max } \times \begin{cases}\exp \left[-\left(t-t_{1}\right)^{2} / \tau^{2}\right], & t<t_{1} \\ 1, & t_{1} \leqslant t \leqslant t_{2} \\ \exp \left[-\left(t-t_{2}\right)^{2} / \tau^{2}\right], & t_{2}<t\end{cases}
$$

which mimics the experimental protocol [29]. The pulse duration is set by the start and end times, which are fixed at $t_{1}=8.39 \mathrm{~ms}$ and $t_{2}=13.63 \mathrm{~ms}$, the switching time is given by $\tau=2.2 \mathrm{~ms}$, and all values are motivated by the experimental boundary conditions (see the SM for details [60]). The amplitude $U_{0}^{\max }$ is the important control parameter that is used to drive the system from the linear into the nonlinear regime. The function $U_{0}(t)$ is shown as black dashed curves in Figs. 4(a)-4(c). field

For better interpretation of our results, we introduce the negative derivative of the displacement

$$
-U_{x}(x, t)=-\frac{\partial U(x, t)}{\partial x}
$$

which is a dimensionless quantity that is, according to Eq. (21), a measure of the relative local lipid area change or compression. We show in Fig. 3 numerically calculated solutions of the nonlinear fractional wave equation (23) for three different driving amplitudes $U_{0}^{\max }$ as solid colored lines. The equilibrium area per lipid is taken as $\bar{a}=88.4 \AA^{2}$, corresponding to a monolayer that is quite far from the minimum in the area modulus (see Fig. 2). Figures 3(a)-3(c) show the displacement $U(x, t)$ as a function of position $x$ for a few different fixed times. Figures 3(d)-3(f) show the corresponding compression profiles $-U_{x}$, defined in Eq. (28), which are just the negative spatial derivatives of the displacement profiles in Figs. 3(a)-3(c). The three driving amplitudes $U_{0}^{\max }$ are chosen so as to illustrate the effects of the nonlinear term in Eq. (23). For the smallest driving amplitude $U_{0}^{\max }=10^{-3} \mathrm{~mm}$, the numerically calculated profiles in Figs. 3(a) and 3(d) (solid colored lines) perfectly agree with the analytic solutions of the linearized fractional wave equation (17) (dashed colored lines) (see the SM for details on this comparison [60]). We thus not only see that the numerical algorithm works, but also find that $U_{0}^{\max }=10^{-3} \mathrm{~mm}$ is in the linear regime. For the intermediate driving amplitude $U_{0}^{\max }=0.54$ $\mathrm{mm}$ in Figs. 3(b) and 3(e) one can discern pronounced deviations between the nonlinear numerical results and the linear predictions, so a submillimeter driving amplitude already moves the system deep into the nonlinear regime. For the largest driving amplitude $U_{0}^{\max }=1.85 \mathrm{~mm}$ in Figs. 3(c) and 3 (f) we see that the nonlinear equation predicts wave shapes that are completely different from the linear scenario; in particular, the compression profiles in Fig. 3(f) exhibit rather sharp fronts.

In Fig. 4 we show results for the same parameters, now plotted as a function of time $t$ and for a few different values of the fixed separation $x$ from the source of excitation located at $x=0$. This way of presenting the data is in fact quite close to how nonlinear surface waves are studied experimentally [29]. Figures 4(a)-4(c) again show the displacement profiles $U(x, t)$, while Figs. 4(d)-4(f) show the corresponding compression profiles $-U_{x}(x, t)$. The black curves for $x=0$ show the excitation pulse that is applied at the boundary $x=0$, which drives the surface wave. Again, we see that for 

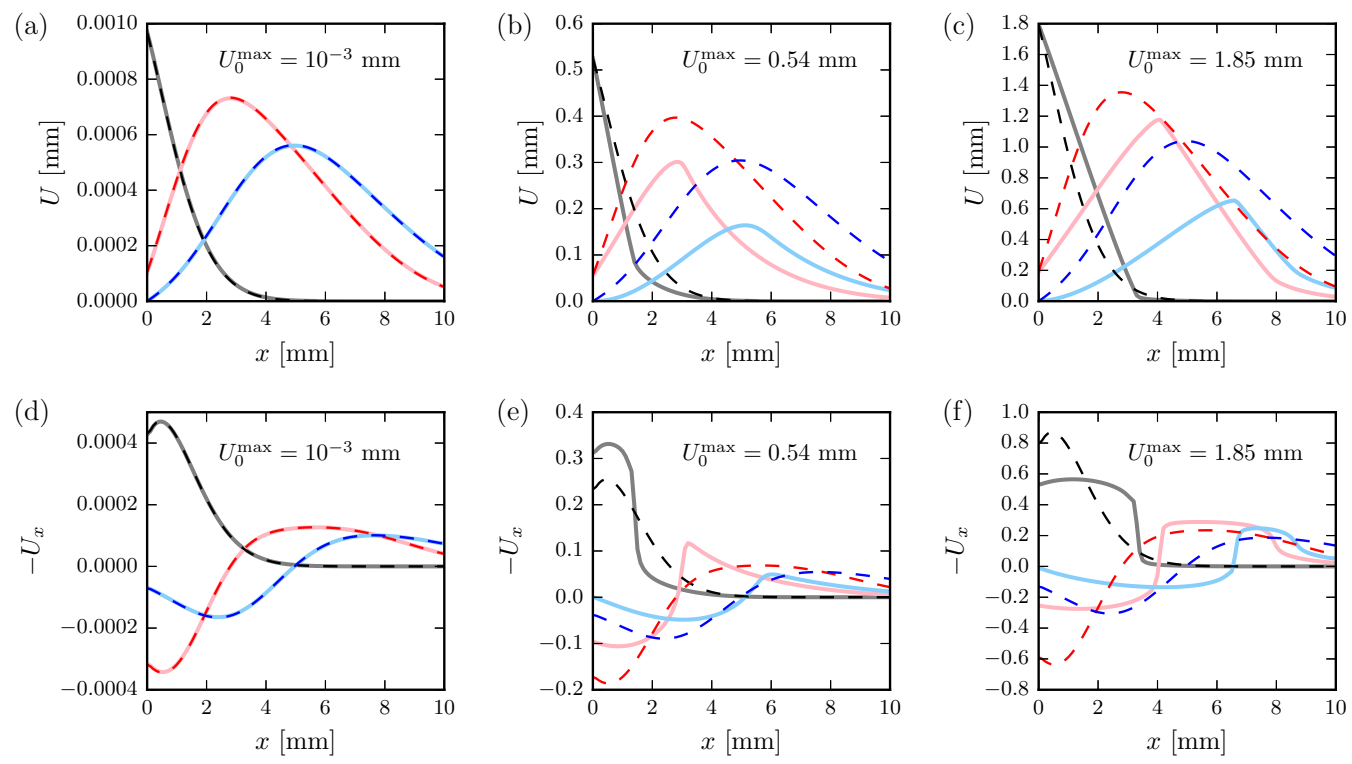

$$
\begin{array}{r}
\text { Numerical }-t=8 \mathrm{~ms}-t=17 \mathrm{~ms}-t=20 \mathrm{~ms} \\
\text { Analytical (linear) - }-t=8 \mathrm{~ms}--t=17 \mathrm{~ms}--t=20 \mathrm{~ms}
\end{array}
$$

FIG. 3. Displacement and compression profiles as a function of position $x$ for a few different fixed times. Results are shown for three different driving amplitudes $U_{0}^{\max }$ as indicated in the legends and for a fixed equilibrium area per lipid of $\bar{a}=88.4 \AA^{2}$. Solid lines are obtained by numerical solution of the nonlinear fractional wave equation (23). Dashed lines denote analytical solutions of the linear fractional wave equation (17) with $\rho_{2 \mathrm{D}}=0$. Compression profiles in (d)-(f) are calculated according to Eq. (28).

the smallest driving amplitude $U_{0}^{\max }=10^{-3} \mathrm{~mm}$ in Figs. 4(a) and 4(d) the agreement between the numerical profiles (solid colored lines) and the analytic linear solutions (dashed colored lines) is perfect. The wave shape, which at the boundary $x=0$ resembles a pulse with rather sharp flanks, changes into a much smoother function as one moves away from the driven boundary. Distinct deviations between nonlinear and linear predictions occur for larger values of $U_{0}^{\max }$, as shown in Figs. 4(b) and 4(e) and Figs. 4(c) and 4(f).

Based on $-U_{x}(x, t)$, we consider two observables that are directly measured in our experiments. The first is the maximal local compression at a fixed separation $x$ from the excitation source

$$
-\frac{\Delta a^{\min }(x)}{\bar{a}}=-\min _{t}\left\{U_{x}(x, t)\right\} .
$$

This maximal compression is in the experiments measured by the locally resolved fluorescence of pressure-sensitive dyes that are incorporated into the monolayer [77], as will be further explained below.

The other important observable is the wave speed, defined by

$$
c(x)=\frac{x}{t_{\min }(x)-t_{1}+\tau},
$$

where $t_{\min }(x)$ is the time at which the maximal compression with a value of $-\Delta a^{\min } / \bar{a}$ arrives at position $x$ (see Fig. 5 for a schematic illustration). Note that the denominator in Eq. (30) is a measure of the difference of the time at which the boundary excitation $U_{0}(t)$ has risen to $1 / e$ of its maximal value, which happens at $t=t_{1}-\tau$, and the time at which the monolayer is maximally compressed at position $x$. 

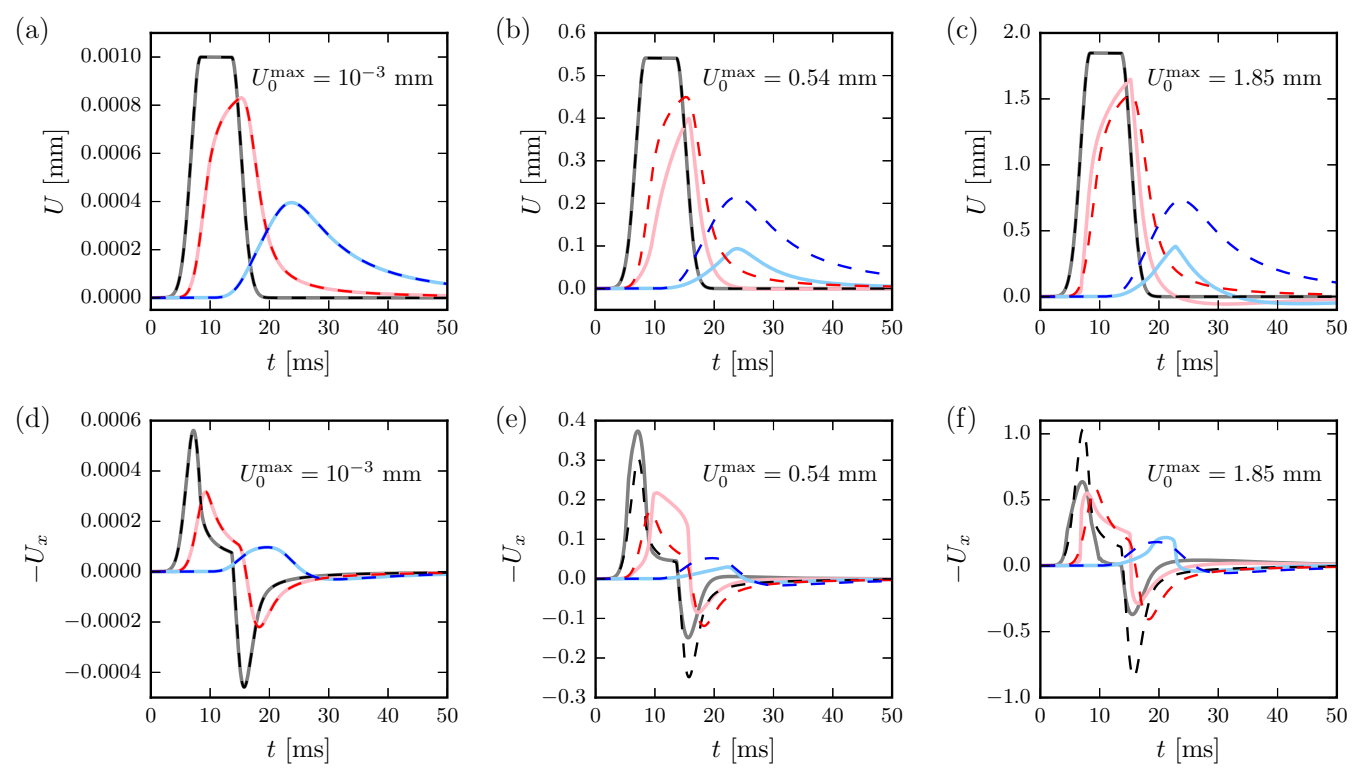

$$
\begin{array}{r}
\text { Numerical }-x=0 \mathrm{~mm}-x=2 \mathrm{~mm}-x=8.4 \mathrm{~mm} \\
\text { Analytical (linear) - }-x=0 \mathrm{~mm}-x=2 \mathrm{~mm}--x=8.4 \mathrm{~mm}
\end{array}
$$

FIG. 4. Displacement and compression profiles as a function of time $t$ for a few different fixed separations $x$ from the driven boundary. Results are shown for three different driving amplitudes $U_{0}^{\max }$ as indicated in the legends and for a fixed equilibrium area per lipid of $\bar{a}=88.4 \AA^{2}$. Solid lines are obtained by numerical solution of the nonlinear fractional wave equation (23). Dashed lines denote analytical solutions of the linear fractional wave equation (17) with $\rho_{2 \mathrm{D}}=0$. The dashed black curves for $x=0$ show the driving function $U_{0}(t)$ that is imposed as a boundary condition. Compression profiles in (d)-(f) are calculated according to Eq. (28).

In Fig. 6(b) we show the maximal compression $-\Delta a^{\min } / \bar{a}$ as defined in Eq. (29) as a function of the driving amplitude $U_{0}^{\max }$ at a fixed separation $x=8.4 \mathrm{~mm}$ from the driving boundary, which is the same separation as used in the experiments [29]. Different colors correspond to different values of the equilibrium area per lipid $\bar{a}$; all employed values of $\bar{a}$ are denoted in Fig. 6(a) by spheres with matching colors, superimposed with the quadratic fit for the monolayer elastic modulus $K_{2 \mathrm{D}}$ used in the calculations. For small excitation amplitudes $U_{0}^{\max }$ linear behavior is obtained and the maximal compression $-\Delta a^{\min } / \bar{a}$, which in Fig. 6(b) is divided by the driving amplitude $U_{0}^{\max }$, exhibits a plateau.

As $U_{0}^{\max }$ is increased, nonlinear effects are noticeable, meaning that the ratio $-\Delta a^{\min } / \bar{a} U_{0}^{\max }$ depends on $U_{0}^{\max }$. This nonlinear behavior depends sensitively on the equilibrium area per lipid $\bar{a}$ and in particular on whether $\bar{a}$ is larger or smaller than $a_{0} \approx 75 \AA^{2}$ for which the elastic modulus $K_{2 \mathrm{D}}$ is minimal. For $\bar{a}<a_{0}$ nonlinear effects lead to a monotonic increase of $-\Delta a^{\mathrm{min}} / \bar{a} U_{0}^{\max }$ with rising $U_{0}^{\max }$ [see the violet curve for $\bar{a}=70 \AA^{2}$ in Fig. 6(b)]. In contrast, for $\bar{a}>a_{0},-\Delta a^{\min } / \bar{a} U_{0}^{\max }$ first decreases and then shows a sudden jump as $U_{0}^{\max }$ increases [see the red curve for $\bar{a}=90 \AA$ in Fig. 6(b)]. The latter behavior is close to what has been seen experimentally [29].

The dependence of the wave speed $c$ in Fig. 6(c) on the excitation amplitude shows an even more pronounced nonlinear behavior. For $\bar{a}<a_{0}$ nonlinear effects lead to a monotonic and smooth increase of the wave speed as a function of the driving amplitude $U_{0}^{\max }$, while for $\bar{a}>a_{0}$ the speed decreases slightly and then abruptly increases at a threshold amplitude of about $U_{0}^{\max }=2 \mathrm{~mm}$. These excitation amplitudes are easily reached experimentally and are thus relevant to the experimentally observed nonlinear effects, as will be discussed later.

Since the nonlinear effects in our theory are introduced via taking into account local variations of the area modulus $K_{2 \mathrm{D}}$, they can be rationalized by analyzing how the linear Lucassen relation 


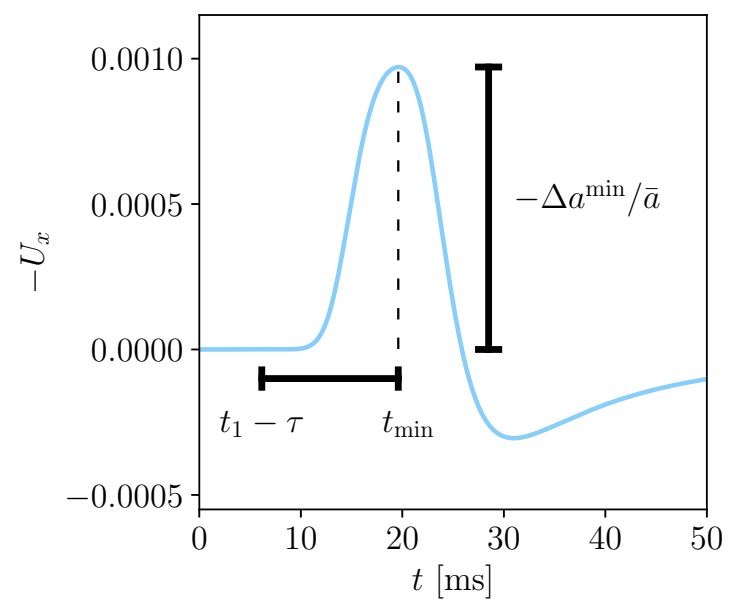

FIG. 5. Illustration of the time it takes to observe maximal compression at a fixed position. The blue curve shows the compression field observed at $x=8.4 \mathrm{~mm}$ for a driving amplitude $U_{0}^{\max }=10^{-3} \mathrm{~mm}$. The vertical black line indicates the maximal compression $-\Delta a^{\mathrm{min}} / \bar{a}$ at $x=8.4 \mathrm{~mm}$, i.e., the value of the maximum of the blue curve. The horizontal black line indicates the time difference between the boundary condition rising to $1 / e$ of its maximal value, which happens at time $t_{1}-\tau \approx 7 \mathrm{~ms}$, and the time when the maximal compression is observed at $x=8.4 \mathrm{~mm}, t_{\min } \approx 20 \mathrm{~ms}$. The difference between these times is used to calculate the wave speed in Eq. (30).

depends on $K_{2 \mathrm{D}}$. Within the linear Lucassen theory, the characteristic length that characterizes the damping along the wave propagation direction is given by

$$
\lambda_{\|}=\frac{1}{\operatorname{Im}(k)} \sim \sqrt{K_{2 \mathrm{D}}}
$$
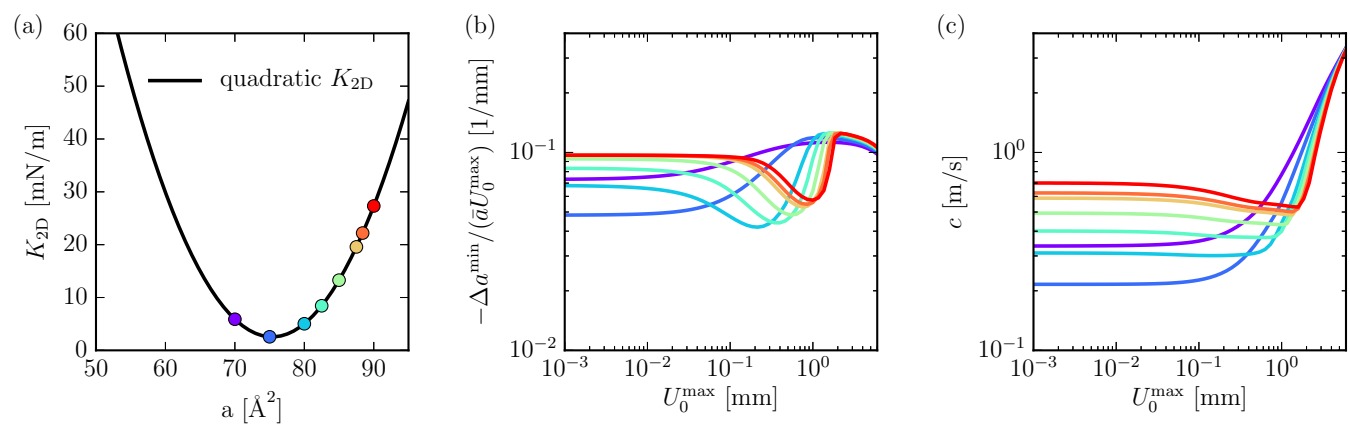

$$
\begin{aligned}
& -\bar{a}=70.0 \AA^{2}-\bar{a}=80.0 \AA^{2}-\bar{a}=85.0 \AA^{2}-\bar{a}=88.4 \AA^{2} \\
& -\bar{a}=75.0 \AA^{2}-\bar{a}=82.5 \AA^{2}-\bar{a}=87.5 \AA^{2}-\bar{a}=90.0 \AA^{2}
\end{aligned}
$$

FIG. 6. Numerical nonlinear results. (a) The black line shows the quadratic fit to the elastic modulus shown in Fig. 2. Colored dots are the different initial areas per lipid $\bar{a}$ used for generating plots (b) and (c). (b) and (c) Numerical results for the boundary-value problem given by Eqs. (23)-(26), with the boundary condition given by Eq. (27) and the quadratic $K_{2 \mathrm{D}}$ shown in (a), for different initial areas per molecule $\bar{a}$ : (b) maximal compression $-\Delta a^{\min } / \bar{a}$ at a distance $x=8.4 \mathrm{~mm}$ from the excitation source, calculated using Eq. (29) and divided by $U_{0}^{\max }$, and (c) the corresponding wave velocity $c$ according to Eq. (30). 
while the phase velocity follows as

$$
c_{\|}=\frac{\omega}{\operatorname{Re}(k)} \sim \sqrt{K_{2 \mathrm{D}}}
$$

where we used the result in Eq. (14) for the wave number $k(\omega)$. According to Eqs. (31) and (32), a larger area modulus $K_{2 \mathrm{D}}$ thus leads not only to a larger decay length $\lambda_{\|}$, but also to a larger phase velocity $c_{\|}$. For an initial area $\bar{a}=70 \AA^{2}$, in the compressive part of the pulse, i.e., where $-U_{x}>0$, the monolayer is compressed and thus characterized by a smaller local area $a<\bar{a}$. From Fig. 6(a) it becomes clear that since $\bar{a}$ is located to the left of the minimum at $a_{0}$, this compression increases the local area modulus. Thus, according to Eqs. (31) and (32), for $\bar{a}<a_{0}$, nonlinear effects are expected to increase the range and the speed of the surface waves, as indeed seen in Fig. 6.

For initial areas $\bar{a}>a_{0}$, on the other hand, a small local compression will decrease $K_{2 \mathrm{D}}$, and only beyond a certain threshold driving amplitude the regime $a<a_{0}$, where $K_{2 \mathrm{D}}$ increases upon further local compression, will be reached. We can thereby explain the nonmonotonic behavior of the maximal compression and the wave speed seen in the numerical data in Figs. 6(b) and 6(c) in a simple manner. In physical terms, the minimum in range and velocity for $\bar{a}>a_{0}$ occurs when nonlinear compression effects are large enough to locally drive the membrane into the minimum in the area modulus $K_{2 \mathrm{D}}$ located at $a_{0}$.

\section{COMPARISON WITH EXPERIMENTAL DATA}

Nonlinear surface waves in a DPPC monolayer have been recently discovered experimentally $[29,73,77]$. In the experimental setup, a DPPC monolayer that contains a small amount of pressuresensitive fluorophores is spread at the air-water interface. A razor blade is placed on top of the interface so that it touches the monolayer at a line, consistent with the harmonic wave ansatz (8) and (9) upon which our theory is based, which assumes that the displacement field decays exponentially for $z<0$, i.e., away from the interface. A piezoelement is used to drive the blade laterally and thereby to compress the monolayer at one end. The excitation pulse shape resembles the smoothed rectangular pulse defined in Eq. (27). At a fixed separation $x=8.4 \mathrm{~mm}$ from the razor blade a fast camera records the fluorescence resonance energy transfer (FRET) efficiency of the fluorophores as a function of time. Using an independent measurement of the FRET efficiency as a function of the area per lipid for an equilibrium isothermal compression of a DPPC monolayer, the recorded time-dependent FRET efficiency is converted into the time-dependent area per lipid $a(t)$, as described in detail before [73]. Waves are excited using different driving voltage amplitudes $V_{0}$ of the piezoelement; for each value of $V_{0}$ the FRET efficiency as a function of time is recorded and converted to yield the compression $\Delta a(t)=a(t)-\bar{a}$. From the maximum of $\Delta a(t)$ the maximal compression $-\Delta a^{\min }$ at a separation $x=8.4 \mathrm{~mm}$ and the time shift $t_{\min }$ at which this maximal compression occurs are calculated [73]. Figure 7(a) shows the experimental results for the relative maximal compression $-\Delta a^{\mathrm{min}} / \bar{a}$ (red spheres) as a function of the piezodriving potential $V_{0}$. The data show a steep increase at a threshold excitation amplitude and level off at a compression of roughly $-\Delta a^{\mathrm{min}} / \bar{a} \approx 0.2$. The experimental wave velocity (red spheres) in Fig. 7(b) slightly increases with rising driving voltage and is on the order of $c \approx 0.35 \mathrm{~m} / \mathrm{s}$.

To compare with our theoretical results we evaluate Eqs. (23)-(26) at an equilibrium lipid area $\bar{a}=$ $88.4 \AA^{2}$, which corresponds to the experimental equilibrium surface pressure $\pi=4.3 \mathrm{mN} / \mathrm{m}$ (see Fig. 2). For different values of the excitation amplitude $U_{0}^{\max }$ we calculate the maximal compression and the wave velocity $c$ at a separation $x=8.4 \mathrm{~mm}$ using Eqs. (29) and (30). Figure 7 shows that our theory (blue data points connected by lines) is in reasonable agreement with the experiments; the only adjustable parameter in the comparison is a rescaling of the driving amplitude $U_{0}^{\max }$, which is necessary since the piezovoltage cannot precisely be converted to the oscillation amplitude of the razor blade. The theoretical maximal relative compression $-\Delta a^{\mathrm{min}} / \bar{a}$ shows a quite sharp increase of the relative compression from around $-\Delta a^{\min } / \bar{a} \approx 0.1$ to $-\Delta a^{\min } / \bar{a} \approx 0.2$, while the experimental data seem to increase from $-\Delta a^{\min } / \bar{a} \approx 0$ to $-\Delta a^{\min } / \bar{a} \approx 0.2$. In both theory and experiment, 

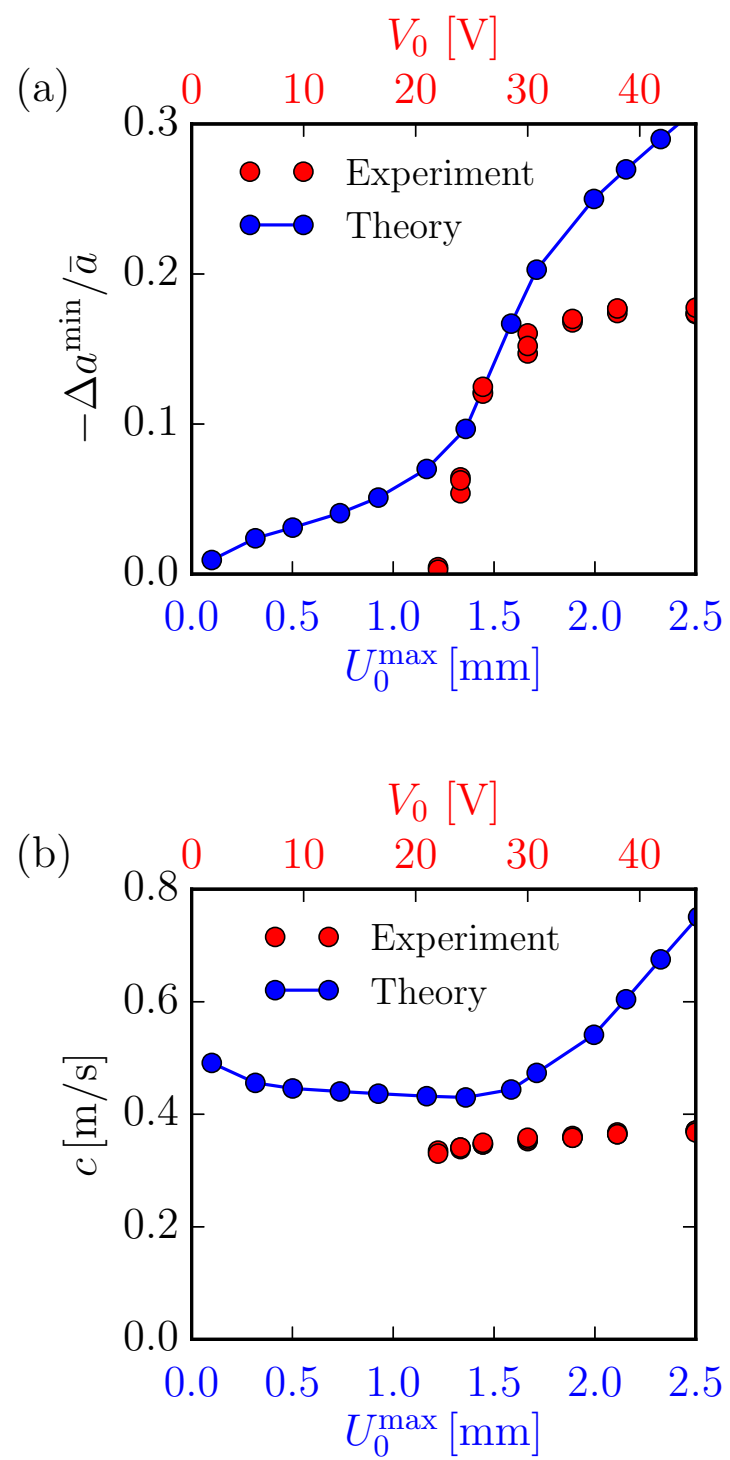

FIG. 7. Comparison of numerical and experimental results at a distance $x=8.4 \mathrm{~mm}$ from the excitation source as a function of the excitation amplitude. The numerical data are obtained by solution of the boundaryvalue problem given by Eqs. (23)-(26), with the boundary condition given by Eq. (27) and the quadratic elastic modulus $K_{2 \mathrm{D}}$ shown in Fig. 2, and various driving amplitudes $U_{0}^{\max }$. The initial area per lipid $\bar{a}=88.4 \AA^{2}$ corresponds to an initial pressure $\pi=4.3 \mathrm{mN} / \mathrm{m}$ (cf. Fig. 2). Observables are calculated using Eqs. (29) and (30), with $x=8.4 \mathrm{~mm}$. The experimental data are obtained by exciting waves in a DPPC monolayer on a Langmuir trough filled with water using various driving voltages $V_{0}$ and measuring the FRET efficiency of pressure sensitive fluorophores at distance $x=8.4 \mathrm{~mm}$ away from the excitation source [29,73]. The equilibrium surface pressure of the DPPC monolayer is $\pi=4.3 \mathrm{mN} / \mathrm{m}$.

at the threshold driving amplitude, a slight increase in the wave velocity is obtained; the value of the wave velocity is quite similar in experiments and theory. This is remarkable, since no freely adjustable parameter is present in the theory. 
One possible reason for the deviations between theory and experiments is that our theoretical model employs the isothermal elastic modulus extracted from the equilibrium pressure isotherm shown in Fig. 2. This is an approximation, since the temperature is not expected to be strictly constant during the wave propagation, as mentioned before [60]. Indeed, it is well known that isotherms obtained from compressing a monolayer depend on the compression speed used [78], that the slowest relaxation modes in a lipid monolayer are on time scales comparable to those of the wave oscillation time [79,80], and furthermore that the viscoelastic properties of lipid membranes close to phase transitions can show anomalous behavior like an effective negative surface viscosity [26]. Thus, the elastic modulus relevant for the nonequilibrium phenomenon of a propagating large-amplitude surface wave might differ significantly from the isothermal elastic modulus characterizing the quasistatic monolayer compression. Turning this around, our theory might in fact be used to shed light on the transition of monolayer elasticity from the isothermal to the adiabatic regime, as will be explained below.

\section{CONCLUSION}

We have derived a fractional wave equation for a compressible surface wave on a viscous liquid from classical hydrodynamic equations. This fractional wave equation has a simple physical interpretation in terms of the frequency-dependent penetration depth of the surface wave into the liquid subphase. Our derivation complements previous approaches where fractional wave equations were obtained by invoking response functions with fractional exponents [48-52] and constitutes a derivation of a fractional wave equation from first physical principles. Therefore, on a fundamental level, our theory sheds light on how fractional wave behavior emerges from the viscous coupling of an interface to the embedding bulk medium.

For the explicit system of a monolayer at the air-water interface, nonlinear behavior emerges naturally since large monolayer compression changes the local monolayer compressibility. Our theory describes the experimentally observed nonlinear acoustic wave propagation in a DPPC monolayer without adjustable fit parameters. In particular, the all-or-nothing response for the maximal compression of a monolayer as a function of the driving amplitude is reproduced and explained by the fact that the acoustic wave locally drives the monolayer through a smeared-out phase transition.

Our theory reveals the origin of nonlinear behavior of pressure waves in compressible monolayers, which is fundamentally different from the nonlinear mechanism for action potential propagation. The connection between these two phenomena, which experimentally are always measured together, have fascinated researchers from different disciplines for a long time [28-31,81].

Our theory might also be used to extract nonequilibrium mechanical properties of biomembranes: Experimental monolayer compressibilities depend on the compression speed employed in the measurement [78]; consequently, the elastic modulus that enters the Lucassen wave theory is neither strictly isothermal nor adiabatic [60]. Our theory could via inversion be used to extract the elastic modulus from experimentally measured surface wave velocities and thereby help to bridge the gap from isothermal membrane properties to adiabatic membrane properties, which is relevant for membrane kinetics.

More specifically, our theory might be able to shed light on the origin of effective negative surface viscosities of lipid membranes extracted from surface wave experiments [26]. In these experiments, linear surface wave theories are employed to analyze experimental data and a negative surface viscosity in a linear theory could be the signature of nonlinear effects that enhance wave propagation, as indeed observed in our nonlinear theory.

\section{ACKNOWLEDGMENTS}

We thank the Deutsche Forschungsgemeinschaft for support via Grant No. SFB 1114 in Project No. C02, "Water diffusion at biological molecules and interfaces: Bridging stochastic and hydrodynamic descriptions." Discussions with A. Mielke are gratefully acknowledged. 
[1] M. Rabaud and F. Moisy, Ship Wakes: Kelvin or Mach Angle? Phys. Rev. Lett. 110, 214503 (2013).

[2] A. Likar and N. Razpet, Towards the Kelvin wake and beyond, Am. J. Phys. 81, 245 (2013).

[3] Wave Fields in Real Media: Wave Propagation in Anisotropic, Anelastic, Porous and Electromagnetic Media, edited by J. M. Carcione (Elsevier, Amsterdam, 2007), Vol. 38.

[4] N. M. Shapiro, High-resolution surface-wave tomography from ambient seismic noise, Science 307, 1615 (2005).

[5] P. Hess, Surface acoustic waves in materials science, Phys. Today 55(3), 42 (2002).

[6] A. Ben-Menahem and S. J. Singh, Seismic Waves and Sources (Springer, New York, 1981).

[7] A. D. D. Craik, The origins of water wave theory, Annu. Rev. Fluid Mech. 36, 1 (2004).

[8] W. Thomson, Ripples and waves, Nature (London) 5, 1 (1871).

[9] L. Rayleigh, On waves propagated along the plane surface of an elastic solid, Proc. London Math. Soc. s1-17, 4 (1885).

[10] G. B. Airy, Tides and waves, Enc. Metropolitana 3 (1841).

[11] P. K. Currie, M. A. Hayes, and P. O’Leary, Viscoelastic Rayleigh waves, Q. Appl. Math. 35, 35 (1977).

[12] P. K. Currie and P. O’Leary, Viscoelastic Rayleigh waves II, Q. Appl. Math. 35, 445 (1978).

[13] R. D. Borcherdt, Viscoelastic Waves Layered Media (Cambridge University Press, Cambridge, 2009).

[14] J. L. Harden, H. Pleiner, and P. A. Pincus, Hydrodynamic surface modes on concentrated polymer solutions and gels, J. Chem. Phys. 94, 5208 (1991).

[15] J. L. Harden and H. Pleiner, Hydrodynamic modes of viscoelastic polymer films, Phys. Rev. E 49, 1411 (1994).

[16] J. Lucassen, Longitudinal capillary waves. Part 2.-Experiments, Trans. Faraday Soc. 64, 2230 (1968).

[17] J. Lucassen, Longitudinal capillary waves. Part 1.-Theory, Trans. Faraday Soc. 64, 2221 (1968).

[18] E. H. Lucassen-Reynders and J. Lucassen, Properties of capillary waves, Adv. Colloid Interface Sci. 2, 347 (1970).

[19] J. Lucassen and M. Van Den Tempel, Longitudinal waves on visco-elastic surfaces, J. Colloid Interface Sci. 41, 491 (1972).

[20] F. Behroozi, B. Lambert, and B. Buhrow, Noninvasive measurement of viscosity from damping of capillary waves, ISA Trans. 42, 3 (2003).

[21] F. Monroy and D. Langevin, Direct Experimental Observation of the Crossover from Capillary to Elastic Surface Waves on Soft Gels, Phys. Rev. Lett. 81, 3167 (1998).

[22] T. Chou, Band structure of surface flexural-gravity waves along periodic interfaces, J. Fluid Mech. 369, 333 (1998).

[23] F. Behroozi, J. Smith, and W. Even, Stokes dream: Measurement of fluid viscosity from the attenuation of capillary waves, Am. J. Phys. 78, 1165 (2010).

[24] C. Cinbis and B. T. Khuri-Yakub, A noncontacting technique for measuring surface tension of liquids, Rev. Sci. Instrum. 63, 2048 (1992).

[25] J. Kappler and R. R. Netz, Multiple surface wave solutions on linear viscoelastic media, Europhys. Lett. 112, 19002 (2015).

[26] J. Giermanska-Kahn, F. Monroy, and D. Langevin, Negative effective surface viscosities in insoluble fatty acid monolayers: Effect of phase transitions on dilational viscoelasticity, Phys. Rev. E 60, 7163 (1999).

[27] J. Krägel, J. B. Li, R. Miller, M. Bree, G. Kretzschmar, and H. Möhwald, Surface viscoelasticity of phospholipid monolayers at the air/water interface, Colloid Polym. Sci. 274, 1183 (1996).

[28] J. Griesbauer, S. Bössinger, A. Wixforth, and M. F. Schneider, Propagation of 2D Pressure Pulses in Lipid Monolayers and Its Possible Implications for Biology, Phys. Rev. Lett. 108, 198103 (2012).

[29] S. Shrivastava and M. F. Schneider, Evidence for two-dimensional solitary sound waves in a lipid controlled interface and its implications for biological signalling, J. R. Soc. Interface 11, 20140098 (2014).

[30] A. El Hady and B. B. Machta, Mechanical surface waves accompany action potential propagation, Nat. Commun. 6, 6697 (2015).

[31] T. Heimburg and A. D. Jackson, On soliton propagation in biomembranes and nerves, Proc. Natl. Acad. Sci. USA 102, 9790 (2005).

[32] R. Appali, U. van Rienen, and T. Heimburg, in Advances in Planar Lipid Bilayers and Liposomes, edited by A. Iglic (Elsevier, Amsterdam, 2012), Vol. 16, p. 275. 
[33] M. M. Rvachev, On axoplasmic pressure waves and their possible role in nerve impulse propagation, Biophys. Rev. Lett. 05, 73 (2010).

[34] J. Griesbauer, A. Wixforth, and M. F. Schneider, Wave propagation in lipid monolayers, Biophys. J. 97, 2710 (2009).

[35] L. D. Mosgaard, A. D. Jackson, and T. Heimburg, in Advances in Planar Lipid Bilayers and Liposomes (Ref. [32]), pp. 51-74.

[36] B. Fichtl, S. Shrivastava, and M. F. Schneider, Protons at the speed of sound: Predicting specific biological signaling from physics, Sci. Rep. 6, 22874 (2016).

[37] B. Martinac, M. Buechner, A. H. Delcour, J. Adler, and C. Kung, Pressure-sensitive ion channel in Escherichia coli, Proc. Natl. Acad. Sci. USA 84, 2297 (1987).

[38] B. Coste, J. Mathur, M. Schmidt, T. J. Earley, S. Ranade, M. J. Petrus, A. E. Dubin, and A. Patapoutian, Piezo1 and Piezo2 are essential components of distinct mechanically activated cation channels, Science 330, 55 (2010).

[39] G. H. Kim, P. Kosterin, A. L. Obaid, and B. M. Salzberg, A mechanical spike accompanies the action potential in mammalian nerve terminals, Biophys. J. 92, 3122 (2007).

[40] I. Tasaki, A. Watanabe, R. Sandlin, and L. Carnay, Changes in fluorescence, turbidity, and birefringence associated with nerve excitation, Proc. Natl. Acad. Sci. USA 61, 883 (1968).

[41] I. Tasaki, Mechanical and thermal changes in the Torpedo electric organ associated with its postsynaptic potentials, Biochem. Biophys. Res. Commun. 215, 654 (1995).

[42] B. Coste, B. Xiao, J. S. Santos, R. Syeda, J. Grandl, K. S. Spencer, S. E. Kim, M. Schmidt, J. Mathur, A. E. Dubin, M. Montal, and A. Patapoutian, Piezo proteins are pore-forming subunits of mechanically activated channels, Nature (London) 483, 176 (2012).

[43] S. Sukharev and F. Sachs, Molecular force transduction by ion channels-Diversity and unifying principles, J. Cell Sci. 125, 3075 (2012).

[44] D. J. Acheson, Elementary Fluid Dynamics (Clarendon, Oxford, 1990).

[45] E. V. Vargas, A. Ludu, R. Hustert, P. Gumrich, A. D. Jackson, and T. Heimburg, Periodic solutions and refractory periods in the soliton theory for nerves and the locust femoral nerve, Biophys. Chem. 153, 159 (2011).

[46] F. Mainardi, Fractional Calculus and Waves in Linear Viscoelasticity: An Introduction to Mathematical Models (Imperial College Press, London, 2010).

[47] S. Holm and S. P. Näsholm, Comparison of fractional wave equations for power law attenuation in ultrasound and elastography, Ultrasound Med. Biol. 40, 695 (2014).

[48] M. Caputo, Linear models of dissipation whose Q is almost frequency independent, Ann. Geophys. 19, 383 (1966).

[49] M. G. Wismer, Finite element analysis of broadband acoustic pulses through inhomogenous media with power law attenuation, J. Acoust. Soc. Am. 120, 3493 (2006).

[50] A. Jaishankar and G. H. McKinley, Power-law rheology in the bulk and at the interface: Quasi-properties and fractional constitutive equations, Proc. R. Soc. A 469, 20120284 (2012).

[51] Y. Wang, Generalized viscoelastic wave equation, Geophys. J. Int. 204, 1216 (2016).

[52] S. Holm, S. P. Näsholm, F. Prieur, and R. Sinkus, Deriving fractional acoustic wave equations from mechanical and thermal constitutive equations, Comput. Math. Appl. 66, 621 (2013).

[53] R. C. MacDonald and S. A. Simon, Lipid monolayer states and their relationships to bilayers, Proc. Natl. Acad. Sci. USA 84, 4089 (1987).

[54] J. R. Hazel, Thermal adaptation in biological membranes: Is homeoviscous adaptation the explanation? Annu. Rev. Physiol. 57, 19 (1995).

[55] G. Matsumoto and I. Tasaki, A study of conduction velocity in nonmyelinated nerve fibers, Biophys. J. 20, 1 (1977).

[56] M. Ringkamp, L. M. Johanek, J. Borzan, T. V. Hartke, G. Wu, E. M. Pogatzki-Zahn, J. N. Campbell, B. Shim, R. J. Schepers, and R. A. Meyer, Conduction properties distinguish unmyelinated sympathetic efferent fibers and unmyelinated primary afferent fibers in the monkey, PLoS One 5, e9076 (2010).

[57] F. K. Sanders and D. Whitteridge, Conduction velocity and myelin thickness in regenerating nerve fibres, J. Physiol. 105, 152 (1946). 
[58] D. N. Franz and A. Iggo, Conduction failure in myelinated and non-myelinated axons at low temperatures, J. Physiol. 199, 319 (1968).

[59] A. L. Hodgkin and A. F. Huxley, A quantitative description of membrane current and its application to conduction and excitation in nerve, J. Physiol. 117, 500 (1952).

[60] See Supplemental Material at http://link.aps.org/supplemental/10.1103/PhysRevFluids.2.114804 for detailed derivations and the numerical algorithm.

[61] E. P. Petrov and P. Schwille, Translational diffusion in lipid membranes beyond the Saffman-Delbrück approximation, Biophys. J. 94, L41 (2008).

[62] E. P. Petrov, R. Petrosyan, and P. Schwille, Translational and rotational diffusion of micrometer-sized solid domains in lipid membranes, Soft Matter 8, 7552 (2012).

[63] C.-H. Lee, W.-C. Lin, and J. Wang, All-optical measurements of the bending rigidity of lipid-vesicle membranes across structural phase transitions, Phys. Rev. E 64, 020901 (2001).

[64] N. Delorme and A. Fery, Direct method to study membrane rigidity of small vesicles based on atomic force microscope force spectroscopy, Phys. Rev. E 74, 030901 (2006).

[65] P. A. Kralchevsky, J. C. Eriksson, and S. Ljunggren, Theory of curved interfaces and membranes: Mechanical and thermodynamical approaches, Adv. Colloid Interface Sci. 48, 19 (1994).

[66] M. Caputo, Linear models of dissipation whose Q is almost frequency independent-II, Geophys. J. Int. 13, 529 (1967).

[67] H. Mori, Transport, collective motion, and brownian motion, Prog. Theor. Phys. 33, 423 (1965).

[68] R. Zwanzig, Memory effects in irreversible thermodynamics, Phys. Rev. 124, 983 (1961).

[69] W. M. Haynes, CRC Handbook of Chemistry and Physics, 95th Edition (CRC Press, Boca Raton, FL, 2014).

[70] W. R. Schneider and W. Wyss, Fractional diffusion and wave equations, J. Math. Phys. 30, 134 (1989).

[71] F. Mainardi, The fundamental solutions for the fractional diffusion-wave equation, Appl. Math. Lett. 9, 23 (1996).

[72] L. D. Landau and E. M. Lifshitz, Theory of Elasticity, 3rd ed., Course of Theoretical Physics Vol. 7 (Elsevier, Amsterdam, 2008).

[73] S. Shrivastava, K. H. Kang, and M. F. Schneider, Solitary shock waves and adiabatic phase transition in lipid interfaces and nerves, Phys. Rev. E 91, 012715 (2015).

[74] J. E. Marsden and T. J. R. Hughes, Mathematical Foundations of Elasticity, Dover Civil and Mechanical Engineering Series (Dover Publications, Mineola, NY, 1994).

[75] C. Li, Z. Zhao, and Y. Q. Chen, Numerical approximation of nonlinear fractional differential equations with subdiffusion and superdiffusion, Comput. Math. Appl. 62, 855 (2011).

[76] F. Johansson et al., mpmath: A Python library for arbitrary-precision floating-point arithmetic (version 0.14) (2010), http: //code.google.com/p/mpmath/

[77] S. Shrivastava and M. F. Schneider, Opto-mechanical coupling in interfaces under static and propagative conditions and its biological implications, PLoS ONE 8, e67524 (2013).

[78] K. J. Klopfer and T. K. Vanderlick, Isotherms of dipalmitoylphosphatidylcholine (DPPC) monolayers: Features revealed and features obscured, J. Colloid Interface Sci. 182, 220 (1996).

[79] J. F. Holzwarth, Structure and dynamics of phospholipid membranes from nanoseconds to seconds, in The Enzyme Catalysis Process, edited by A. Cooper, J. L. Houben, and L. C. Chien (Springer US, Boston, MA, 1989) p. 383.

[80] W. W. Van Osdol, R. L. Biltonen, and M. L. Johnson, Measuring the kinetics of membrane phase transitions, J. Biochem. Biophys. Methods 20, 1 (1989).

[81] I. Tasaki, K. Kusano, and P. M. Byrne, Rapid mechanical and thermal changes in the garfish olfactory nerve associated with a propagated impulse, Biophys. J. 55, 1033 (1989). 